\title{
A Geração Distribuída e a Redução de Carbono na Matriz Elétrica Brasileira
}

\author{
Ricardo Abranches Felix Cardoso Junior'; Alessandra Schwertner Hoffmann'; Leonardo de \\ Oliveira Barbosal; Roberta de Azevedo Pires Soares Coutinhol
}

\roberta_coutinho@id.uff.br

1. Universidade Federal Fluminense. Niterói - RJ, Brasil.

\section{Histórico do Artigo:}

Recebido em: 07 de junho de 2020

Resumo: As últimas conferências mundiais de meio ambiente e mudanças climáticas tiveram papel fundamental para difusão e inserção das energias renováveis na matriz elétrica mundial. Nesse contexto, as fontes renováveis de geração distribuída se destacam por apresentarem vantagens tanto no âmbito econômico quanto socioambiental. Este estudo analisa o contexto nacional e internacional da geração distribuída de energia solar fotovoltaica, através da comparação entre eles e proposição de estratégias para uma maior penetração e difusão desta modalidade de geração. É realizada também uma análise temporal da emissão de carbono na matriz elétrica, baseada principalmente na geração termelétrica, e verificada a projeção de emissões com base na evolução da geração distribuída, segundo o PDE 2026. A fim de materializar a redução da emissão, foi parametrizada e calculada a área de Mata Atlântica necessária para sequestrar dado volume de gás carbônico equivalente. Resultados indicaram que, a partir do incremento de potência instalada de 3,6 GW de GD fotovoltaica no horizonte decenal, estima-se evitar a emissão de 337 mil toneladas de $\mathrm{CO}_{2 \mathrm{eq}}$, correspondente a uma área de reflorestamento de aproximadamente 2500 hectares ou 56 vezes o tamanho do território do Vaticano. As análises realizadas visam ampliar os estudos para expansão da participação da geração distribuída solar fotovoltaica na matriz elétrica.

Palavras-chave: Geração distribuída, Geração solar fotovoltaica, Emissões de carbono, Políticas de incentivo.

\section{The Distributed Generation and Carbon Reduction in the Brazilian Electric Matrix}

\begin{abstract}
The latest world conferences on the environment and climate change have played a fundamental role in the diffusion and insertion of renewable energy in the world electrical grid. In this context, renewable sources of distributed generation stand out for presenting advantages in both economic and socio-environmental spheres. This study analyzes the national and international context of distributed generation of solar photovoltaic energy, by comparing them and proposing strategies for greater penetration and diffusion of this modality. A temporal analysis of the carbon emission in the electric grid is also carried out based mainly on thermoelectric generation, and the projection of emissions is verified based on the evolution of distributed generation according to PDE 2026. In order to materialize the emission reduction, it was considered the area in hectares of Atlantic Forest required to sequester a given volume of carbon dioxide equivalent. Based on the analysis of the results, this article concludes that fiscal, economic and financial incentives at regional scales are the main ways to leverage the growth of this modality, as observed in the reference countries. In the environmental aspect, a significant mitigation of the emission of greenhouse gases from this new type of electricity generation was found. Results indicated that, based on the increase in installed capacity of $3.6 \mathrm{GW}$ of photovoltaic GD over the ten-year horizon, it is estimated to avoid the emission of 337 thousand tons of C02eq, corresponding to a reforestation area of approximately 2500 hectares or 56 times the size of the Vatican territory. This paper tried to expand the studies for a greater participation of the solar photovoltaic generation distributed in the electric grid.

Keywords: Distributed generation, Photovoltaic solar generation, Carbon emissions, Incentive policies.
\end{abstract}




\section{Generación Distribuida y Reducción de Carbono en la Matriz Eléctrica Brasileña}

Resumen: Las últimas conferencias mundiales sobre medio ambiente y cambio climático han jugado un papel fundamental en la difusión e inserción de las energías renovables en la red eléctrica mundial. En este contexto, las fuentes renovables de generación distribuida destacan por presentar ventajas tanto en el ámbito económico como socioambiental. Este estudio analiza el contexto nacional e internacional de generación distribuida de energía solar fotovoltaica, comparándolos y proponiendo estrategias para una mayor penetración y difusión de esta modalidad. También se realiza un análisis temporal de la emisión de carbono en la red eléctrica basado principalmente en la generación termoeléctrica, y la proyección de emisiones se verifica en función de la evolución de la generación distribuida según PDE 2026. Para materializar la reducción de emisiones, se realizó consideró el área en hectáreas de bosque atlántico requerida para secuestrar un volumen dado de dióxido de carbono equivalente. Basado en el análisis de los resultados, este artículo concluye que los incentivos fiscales, económicos y financieros a escalas regionales son las principales formas de impulsar el crecimiento de esta modalidad, como se observó en los países de referencia. En el aspecto ambiental, se encontró una mitigación significativa de la emisión de gases de efecto invernadero de este nuevo tipo de generación de electricidad. Los resultados indicaron que, con base en el aumento de la capacidad instalada de 3,6 GW de GD fotovoltaica en el horizonte de diez años, se estima evitar la emisión de 337 mil toneladas de C02eq, correspondiente a un área de reforestación de aproximadamente 2500 hectáreas 056 veces el tamaño del territorio del Vaticano. Este artículo intentó expandir los estudios para una mayor participación de la generación solar fotovoltaica distribuida en la red eléctrica.

Palabras clave: Generación distribuida, Generación solar fotovoltaica, Emisiones de carbono, Políticas de incentivos.

\section{INTRODUÇ̃̃o}

As grandes convenções internacionais sobre o meio ambiente e as mudanças climáticas foram importantes para avanços relacionados à diversificação da matriz energética de diversos países.

Em 2017, a capacidade fotovoltaica solar acumulada atingiu quase 398 GW e gerou mais de 460 TWh, representando cerca de $2 \%$ da produção global de energia (Gráfico 1). Projetos centralizados respondem por pouco mais de $60 \%$ da capacidade instalada total de energia fotovoltaica, com o restante em aplicações distribuídas (residencial, comercial e fora da rede). Nos próximos cinco anos, a energia solar fotovoltaica deverá liderar o crescimento da capacidade de eletricidade renovável, expandindo em quase 580 GW (INTERNATIONAL ENERGY AGENCY, 2019).

Gráfico 1. Geração de energia solar fotovoltaica e capacidade instalada por região, 2017-2023. 


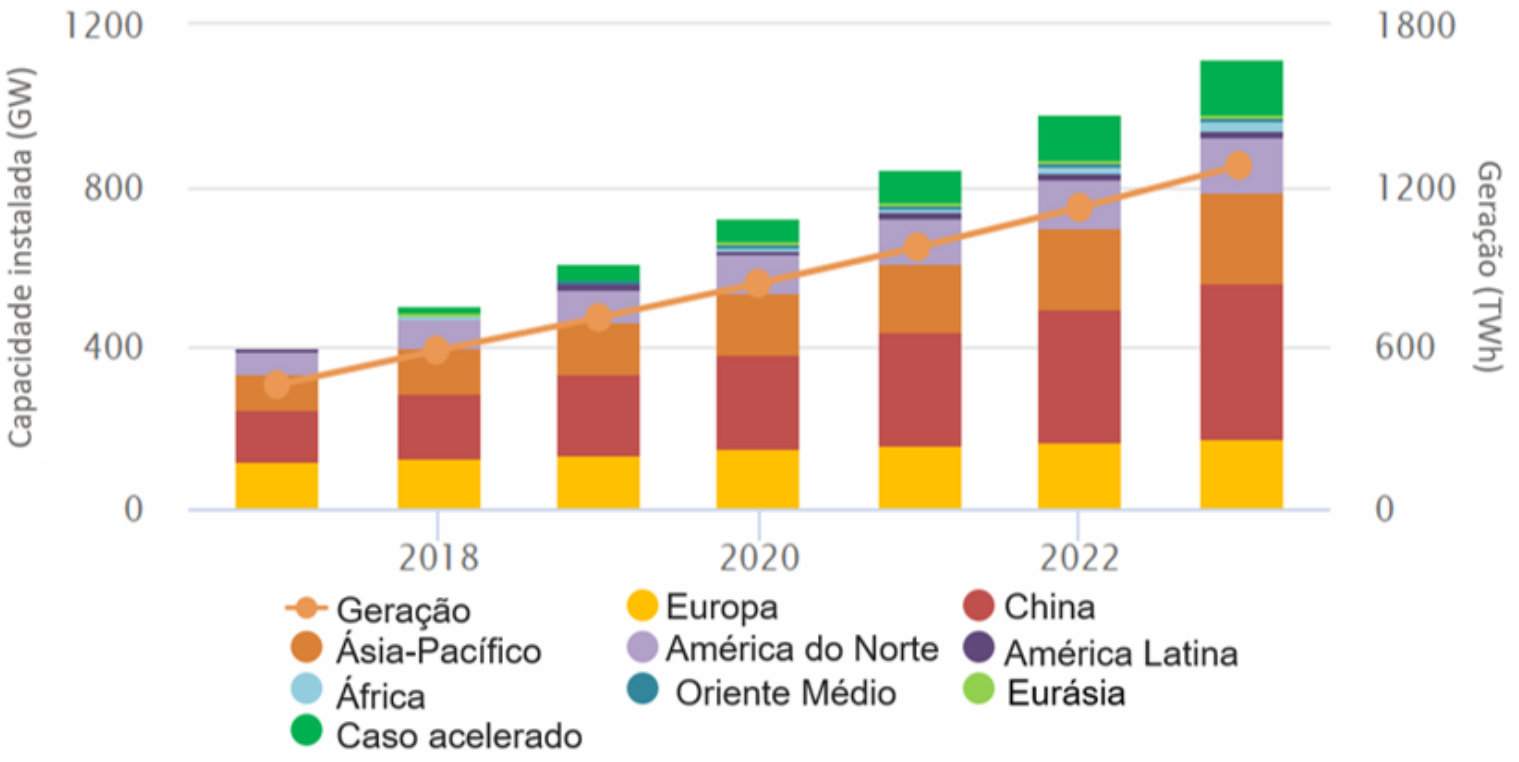

Fonte: IEA, 2018 Adaptado de INTERNATIONAL ENERGY AGENCY, 2019.

Muitos fatores contribuíram para o interesse nessas formas de geração energética e seu crescimento, como por exemplo inovações tecnológicas, a abertura do mercado livre de energia, impactos ambientais para construção de grandes unidades de geração e novas linhas de transmissão, consumidores demandando uma fonte energética cada vez mais confiável e as preocupações com as mudanças climáticas (MINISTÉRIO DO MEI0 AMBIENTE, 2018).

Cabe mencionar que a International Energy Agency (2019) atribui como fator principal para crescimento desse tipo de geração a redução do custo da tecnologia, que implicará em um aumento para 140 GW em 2023, o que a torna mais competitiva globalmente.

Segundo a Iniciativa de Energia Sustentável para Todos das Nações Unidas (SE4ALL, 2014) a tecnologia solar de Geração Distribuída (GD) fotovoltaica contribuirá com $70 \%$ de aumento no acesso das famílias à eletricidade até o ano 2030.

A geração distribuída, que entrou em discussão no Plano Decenal de Expansão de Energia 2026 (PDE 2026) (EMPRESA DE PESQUISA ENERGÉTICA, 2018) como uma nova relação do consumidor com o setor energético, onde este sai de uma posição passiva para se tornar um ator relevante. Mesmo ainda com pouca expressão nacional, a geração distribuída apresenta projeções significativas para próxima década, com expansão da ordem de 80 vezes, como pode observado no Gráfico 2. 
Gráfico 2. Capacidade instalada a partir da geração distribuída por tipologia

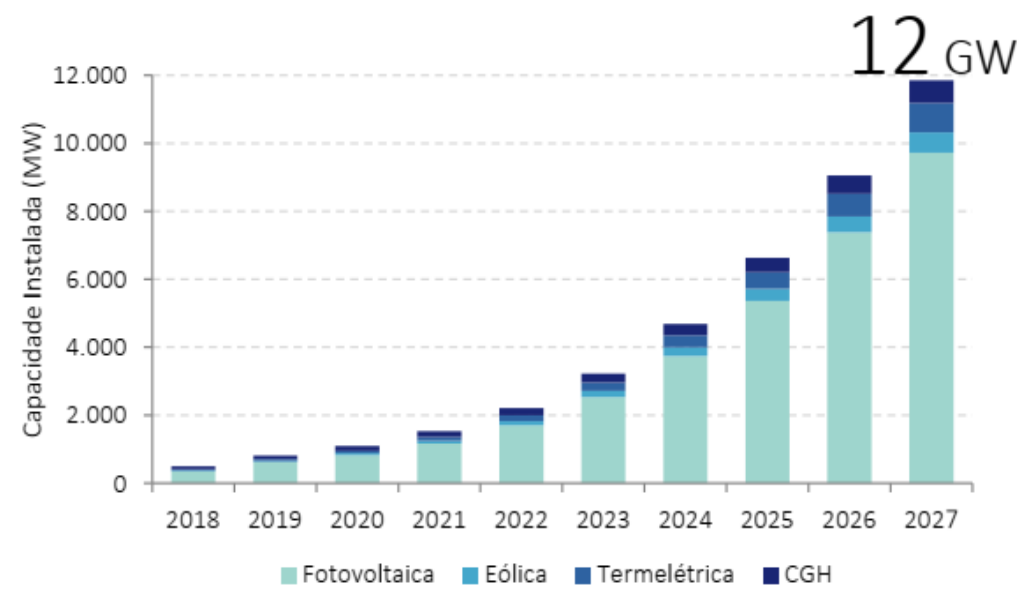

Fonte: EMPRESA DE PESQUISA ENERGÉTICA, 2018.

Assim, o objetivo deste artigo consiste na avaliação do cenário nacional e internacional da geração distribuída fotovoltaica sob a perspectiva de estratégias adequadas para sua promoção, bem como a discussão da projeção desta modalidade na matriz elétrica brasileira em termos de emissão de carbono.

\section{MATERIAIS E MÉTODOS}

A fim de alcançar os resultados esperados, o presente trabalho baseia-se em uma pesquisa exploratória e análise de artigos científicos, marcos regulatórios e projeções do setor elétrico brasileiro, além de pesquisa bibliográfica qualitativa.

No contexto internacional, foram analisadas políticas de incentivo de países protagonistas na modalidade de geração distribuída solar fotovoltaica: Estados Unidos, especificamente na Califórnia, China, Alemanha e Japão.

Foram analisadas as bases de dados brasileiras da ANEEL e Ministério de Ciência e Tecnologia quanto à emissão de carbono da geração térmica.

As projeções para o estabelecimento da GD se apoiaram nos cenários do Plano Decenal de Expansão da Energia (PDE), emitido pela Empresa de Pesquisa de Energia (EPE).

Com base nessas informações, associando-se aos dados de projeção da geração distribuída e termelétrica, foi possível promover análise comparativa, quantificando a evitação da emissão de carbono e, através dela, a mitigação de impacto ambiental associada. 


\section{Geração Distribuída e Tendências}

A geração distribuída é uma fonte de energia elétrica conectada diretamente à rede de distribuição e situada no próprio consumidor (ACKERMANN et. al., 2001).

A geração distribuída está se disseminando de maneira progressiva no país por seus potenciais benefícios ao setor elétrico como um todo como, por exemplo, o adiamento dos investimentos na expansão dos sistemas de transmissão e distribuição de energia, o baixo impacto ambiental do sistema, minimização das perdas energéticas e a diversificação da matriz (ANEEL, 2018).

Entre os fatores de destaque para integração da GD, constam principalmente a redução de emissões de carbono e o menor impacto ambiental associado à geração renovável (HERNÁNDEZ-CALLEJ0 et. al., 2019).

No cenário brasileiro atual, a geração distribuída corresponde a 0,05\% da matriz elétrica, porcentagem ainda irrisória quando comparada com as previsões decenais do setor elétrico brasileiro, que visam 5\% de geração distribuída na matriz elétrica. Observou-se um incremento no número de conexões no ano de 2016, de acordo com dados do Plano Decenal de Expansão de Energia 2026 (PDE 2026), somando 81 MW de potência instalada, aproximadamente 4 vezes superior ao ano anterior. É previsto ainda que no ano de 2027 a potência instalada da geração distribuída fotovoltaica seja de 9,8 GW conforme observamos no Gráfico 3, que demonstra a Capacidade instalada verificada e projetada de geração distribuída solar fotovoltaica no Brasil com base nos dados da Agência Nacional de Energia Elétrica, 2018 e Empresa de Pesquisa Energética.

Gráfico 3. Capacidade Instalada de Geração Solar Fotovoltaica.

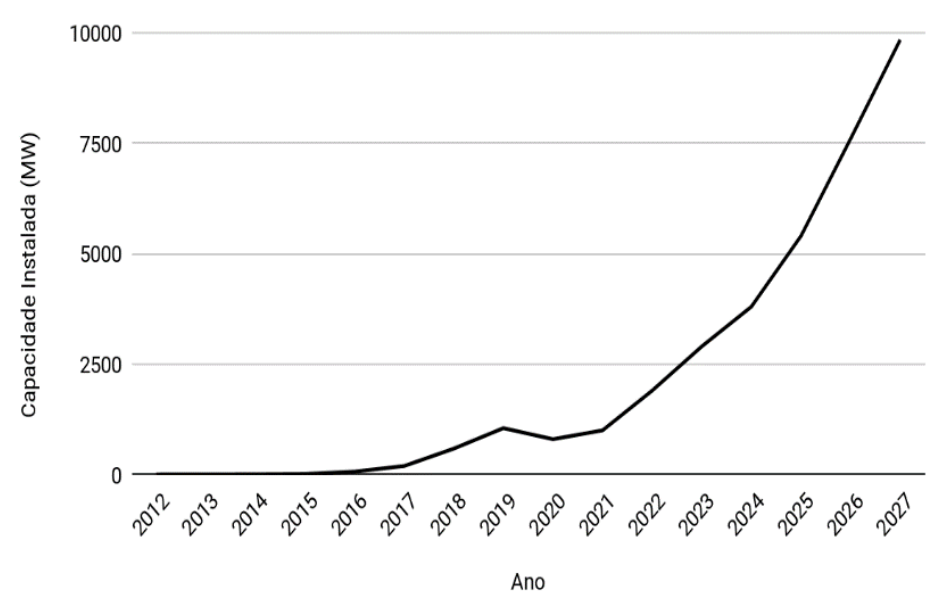


Fonte: Autores Base de dados da AGÊNCIA NACIONAL DE ENERGIA ELÉTRICA, 2018 e EMPRESA DE PESQUISA ENERGÉTICA

Os incentivos fiscais para o estabelecimento de energias renováveis constituem importante forma de promoção desse tipo de tecnologia. A tarifa feed-in, por exemplo, se trata de um mecanismo de políticas públicas que objetiva incrementar investimentos em tecnologias renováveis através de contratos de longo prazo (20 anos) com geradores de energia elétrica, apoiado no custo de geração renovável. Em Portugal, com a remoção de tais incentivos, reduziuse significativamente a instalação de painéis fotovoltaicos em 2015 (PESTANA et al., 2018).

No Brasil, foi criado em 2015 o Programa de Desenvolvimento da Geração Distribuída (ProGD), que tinha como objetivo aumentar a disseminação dos sistemas no país. 0 conjunto destes fatores fez com que a projeção brasileira para o ano de 2018 fosse superada batendo os 1 GW de potência instalada em julho de 2019 (ABSOLAR, 2018).

Conforme é evidenciado no Gráfico 4, a queda dos preços dos painéis atrelada aos outros incentivos mencionados neste trabalho reflete na evolução exponencial do número de sistemas conectados à rede.

Gráfico 4. Quantidade de Sistemas Fotovoltaicos Instalados.

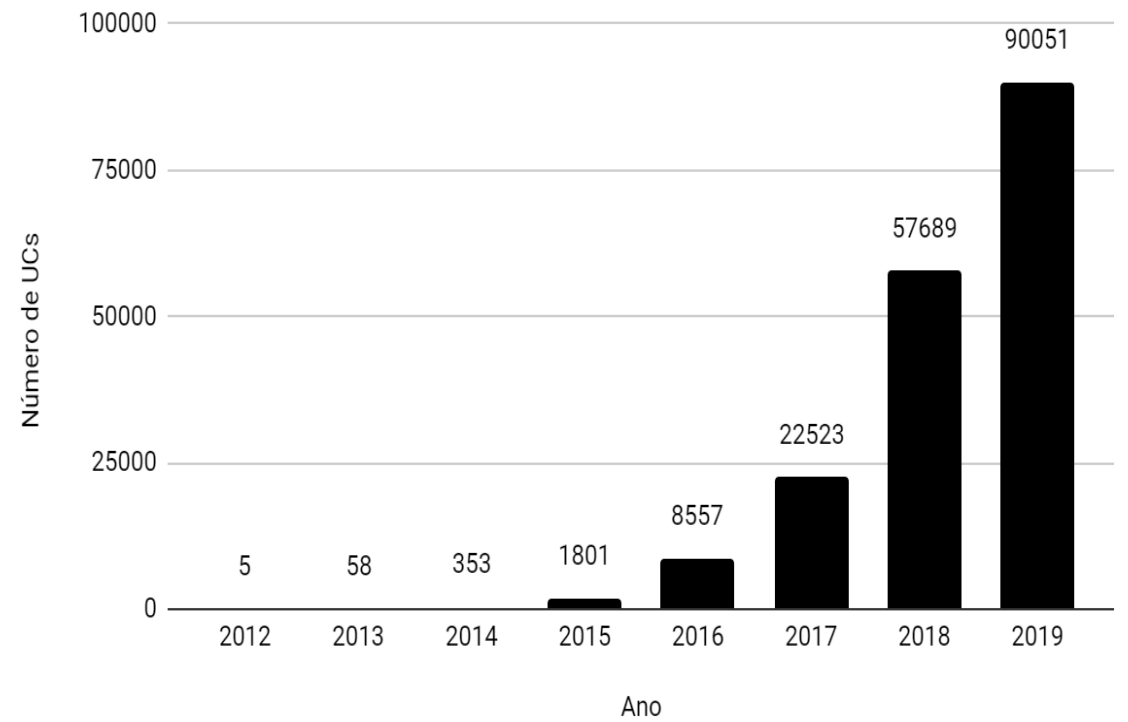

Fonte: AGÊNCIA NACIONAL DE ENERGIA ELÉTRICA, 2019.

\section{Contexto Internacional}

Para verificar a tendência internacional de expansão da geração distribuída e para obter melhores parâmetros de análise e comparação, foram avaliados contextos regulatórios e de investimentos em países como a China, Estados Unidos, Japão e Alemanha. 
Tais países foram escolhidos pela diversidade dos estudos, políticas estratégicas e pela significância da GD na matriz. Em análise da capacidade instalada internacional até 0 ano de 2017, somando-se a geração centralizada solar e a geração distribuída, a China, como maior país gerador de energia elétrica provinda de fonte solar mundial, alcançou $131 \mathrm{GW}$, equivalente a aproximadamente 33\% de toda a geração solar mundial. Na sequência observase os Estados Unidos com potência instalada de 51 GW, o Japão com 49 GW e a Alemanha com 42 GW. Observa-se na Tabela 1 a capacidade instalada em 2017 para cada país.

Tabela 1. 0s 10 principais países para capacidade instalada total em 2017.

\begin{tabular}{ccc}
\hline & País & Capacidade Instalada \\
\hline 1 & China & $131 \mathrm{GW}$ \\
2 & Estados Unidos & $51 \mathrm{GW}$ \\
3 & Japão & $49 \mathrm{GW}$ \\
4 & Alemanha & $42 \mathrm{GW}$ \\
5 & Itália & $19,7 \mathrm{GW}$ \\
6 & Índia & $18,3 \mathrm{GW}$ \\
7 & Reino Unido & $12,7 \mathrm{GW}$ \\
8 & França & $8 \mathrm{GW}$ \\
9 & Austrália & $7,2 \mathrm{GW}$ \\
10 & Espanha & $5,6 \mathrm{GW}$ \\
- & Brasil & $0,2 \mathrm{GW}$ \\
\hline
\end{tabular}

Fonte: Adaptado de IEA Renewables, 2017. ABSOLAR, 2018.

A seguir, foram pontuadas as medidas de destaque adotadas pelos países avaliados.

\begin{abstract}
Alemanha
Na Alemanha, foram identificadas políticas de incentivo como as tarifas feed-in e a queda dos preços dos sistemas. 0 país também estabeleceu a meta de alcançar 40 a 45\% de geração de energia elétrica a partir de fontes renováveis até 2025 e pelo menos $80 \%$ até 2050 , tendo como ator principal dessa transição a disseminação dos recursos energéticos distribuídos, principalmente fotovoltaicos. Em 2000 foi implementada a Lei de Fontes de Energia Renovável (EEG, em alemão), marcando o início de uma nova dinâmica na difusão de energia renovável a partir das tarifas feed-in, que adicionadas ao incentivo à compra de sistemas individuais foram os principais fatores de sucesso da expansão da geração distribuída na Alemanha.
\end{abstract}


Como consequência da EEG, 2008 foi o ano de maior expansão da GD fotovoltaica, em virtude das importações a baixo custo (cerca de 30\% mais barato) dos painéis chineses, representando um marco no cenário da geração distribuída na Alemanha.

Tal planejamento estratégico político/econômico foi fundamental para difusão principalmente dos pequenos sistemas geradores (instalados em telhados), atingindo a capacidade instalada de $23 \mathrm{GW}$, o que representa cerca de $57 \%$ de toda a capacidade fotovoltaica alemã. (DE CASTR0, N.; DANTAS, G., 2017).

\section{Estados Unidos - Califórnia}

Na Califórnia, a participação da microgeração solar fotovoltaica no consumo total de energia é 1,3\%. Até o final do ano de 2016, o regime da Net Energy Metering(NEM -1995 - atual), bem como a lei AB 920 (2009 - atual) foram responsáveis pelos atuais 594.685 sistemas (residenciais e não residenciais) registrados no NEM, o que equivale a uma capacidade de aproximadamente 4,7 GW e corresponde a mais de $90 \%$ da capacidade fotovoltaica total instalada.

Neste contexto, destaca-se a atuação conjunta de três principais políticas: (i) o incentivo à investimentos em projetos de tecnologia em geração renovável; (ii) crédito fiscal para obtenção de novos sistemas de geração distribuída; (iii) e a geração de crédito nas tarifas de contas de luz a partir da injeção de energia elétrica na rede. Como resultado, atualmente a Califórnia é o estado que lidera o processo de difusão da energia elétrica a partir de fonte solar nos Estados Unidos (FRANZ, B., 2016).

\section{Japão}

Diferentemente da Alemanha e dos EUA, o Japão tem como foco a geração em escala diversificada. Especificamente para a geração residencial, são aplicadas as tarifas feed-in, em formato similar ao alemão.

0 país propôs um subsídio financeiro de até 70\% para a implantação de novos sistemas. Rapidamente verificou-se a eficácia do programa tendo em vista o registro da adesão de 250.000 sistemas em 10 anos (tempo de duração do programa). Posteriormente, adotou-se uma postura de financiamento a juros baixos, desacelerando a difusão da tecnologia.

A partir de 2008, com o início da aplicação das tarifas feed-in, o Japão alcançou a marca de 5 GW de capacidade instalada em 3 anos, com a predominância de instalação de sistema residenciais de pequeno porte. Em 2012, os sistemas residenciais chegaram a atingir 58\% da capacidade total, entretanto figurou-se uma saturação do setor residencial. Em 2013, houve uma 
clara inversão da política, que agora aloca os incentivos nos sistemas de maior porte do setor comercial e rural. Os resultados de tais políticas colocaram o Japão entre os três países do mundo com maior capacidade instalada acumulada (MINISTÉRIO DE MINAS E ENERGIA, 2018).

\section{China}

A China é o maior produtor de tecnologia solar do mundo, de acordo com a Agência Internacional de Energia (IEA), e mais de $60 \%$ dos painéis solares do mundo são fabricados na China, justificando por exemplo a implantação de grandes usinas. Estas ações estão alinhadas com as políticas de incentivo e com a diversificação da matriz, a qual ainda conta com quase $2 / 3$ da eletricidade gerada a partir da queima do carvão. Cabe destaque ao estudo de caso da China, que enxerga muito valor na geração distribuída já que é uma forma de aliviar a crise energética do país e promover a reforma do sistema energético (ZHANG, L.; QIN, Q.; WEI, Y., 2019).

A partir do contexto dos países citados, destacam-se políticas que tiveram bons resultados relacionados a popularização dos sistemas de geração distribuída, como as tarifas feed-in, o crédito fiscal no momento de adquirir o sistema e a criação de uma forma de crédito com as distribuidoras quando os sistemas injetam energia na rede.

Apresenta-se a seguir de forma resumida os casos internacionais na Tabela 2, onde se encontram também os resultados em números das políticas discutidas.

Tabela 2. Resumo dos dados internacionais de políticas de incentivos e seus resultados.

\begin{tabular}{|c|c|c|c|}
\hline Local & $\begin{array}{l}\text { Programa de } \\
\text { incentivo }\end{array}$ & Resultado esperado & Investimento \\
\hline Califórnia & $\begin{array}{c}\text { Net Energy Metering } \\
\text { (NEM) - 1995 - atual } \\
\\
\text { Renewable Portfolio } \\
\text { Standard; Business } \\
\text { Energy Investment } \\
\text { Credit; Net Energy } \\
\text { Metering } \\
\text { Lei (AB 920) - 2009 - } \\
\text { atual }\end{array}$ & $\begin{array}{l}\text { - Sistema onde se recebe crédito } \\
\text { na conta de luz conforme a } \\
\text { diferença líquida positiva } \\
\text { entre a geração e o consumo } \\
\text { do sistema } \\
\text { - Expansão anual, em } \\
\text { residências, em torno de } 50 \% \\
\text { - Caso o consumidor exporte } \\
\text { mais eletricidade do que } \\
\text { demande da rede elétrica, } \\
\text { decorrido o período de } 12 \\
\text { meses, ele pode escolher entre } \\
\text { transferir os créditos para o } \\
\text { próximo período de } \\
\text { faturamento ou receber a } \\
\text { compensação pelo superávit } \\
\text { líquido }\end{array}$ & $\begin{array}{l}\text { - 4,7 GW de potência instalada } \\
\text { em } 2016 \\
\text { - US\$ } 540 \text { milhões em projetos } \\
\text { de fontes renováveis entre } \\
\text { 1998-2001 }\end{array}$ \\
\hline
\end{tabular}




\begin{tabular}{|c|c|c|c|}
\hline Brasil & $\begin{array}{l}\text { Resolução Normativa } \\
\text { ANEEL n }{ }^{0} 482 / 2012\end{array}$ & $\begin{array}{l}\text { • Não obteve êxito, baixa adesão } \\
\text { à modalidade }\end{array}$ & $\begin{array}{l}\text { - Baixas Taxas de Juros de linhas } \\
\text { de financiamento }\end{array}$ \\
\hline & 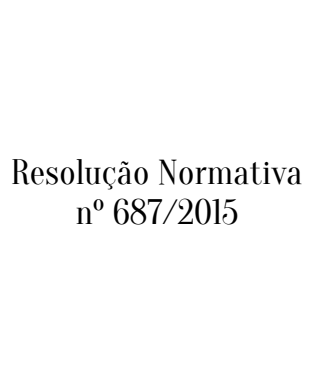 & $\begin{array}{l}\text { - Início do impulso notório da } \\
\text { modalidade que viria a ser } \\
\text { destaque nos anos } \\
\text { subsequentes }\end{array}$ & $\begin{array}{l}\text { - Créditos na fatura, conforme a } \\
\text { produção de energia } \\
\text { - Incentivos fiscais, nos impostos } \\
\text { para importação dos módulos } \\
\text { fotovoltaicos } \\
\text { - Aumento do limite de potência } \\
\text { instalada } \\
\text { - Otimização do tempo para } \\
\text { conexão à rede }\end{array}$ \\
\hline
\end{tabular}

\begin{tabular}{|c|c|c|c|}
\hline \multirow{4}{*}{ Alemanha } & $\begin{array}{c}\text { Programa } 1.000 \\
\text { telhados para } \\
\text { instalação de } \\
\text { sistemas residenciais } \\
\text { (1991) }\end{array}$ & $\begin{array}{l}\text { - } 2200 \text { sistemas e } 5,3 \mathrm{MW} \text { de } \\
\text { capacidade total instalada } \\
(1993)\end{array}$ & $\begin{array}{l}\text { - Auxílio financeiro de até } 70 \% \text { do } \\
\text { custo do investimento }\end{array}$ \\
\hline & $\begin{array}{c}\text { Programa } 100.000 \\
\text { Telhados para } \\
\text { instalação de } \\
\text { sistemas residenciais } \\
\text { (1999) }\end{array}$ & $\begin{array}{l}\text { - Não obteve êxito, devido à } \\
\text { baixa adesão e } \\
\text { consequentemente a meta não } \\
\text { foi atingida }\end{array}$ & $\begin{array}{l}\text { - Linhas de financiamento com } \\
\text { baixas taxas de juros }\end{array}$ \\
\hline & $\begin{array}{l}\text { Lei de Fontes de } \\
\text { Energia Renovável - } \\
\text { EEG (2000-2014) } \\
\text { feed-in } \\
\text { EEG - Reformulada } \\
\text { (2004) - maior } \\
\text { remuneração na feed- } \\
\text { in }\end{array}$ & $\begin{array}{l}\text { - Média anual de crescimento } \\
\text { de } 36 W \text { entre } 2004 \text { e } 2015 \text {, } \\
\text { - Profissionalização da indústria } \\
\text { de renováveis e difusão da } \\
\text { tecnologia entre os setores da } \\
\text { economia }\end{array}$ & $\begin{array}{l}\text { - Tarifa feed-in (TiF) - compra da } \\
\text { energia } \\
\text { - Investimentos que chegaram a } \\
\text { 20,4 bilhões em 2013, segundo o } \\
\text { BMU (Ministério do Meio } \\
\text { Ambiente) }\end{array}$ \\
\hline & $\begin{array}{c}\text { EEG } 2.0- \\
\text { reformulação (2014 - } \\
\text { atual) feed-in } \\
\text { premium }\end{array}$ & $\begin{array}{l}\text { - Redução considerável no } \\
\text { número de sistemas instalados } \\
\text { por ano }\end{array}$ & $\begin{array}{l}\text { - Elegibilidade para receber a TiF } \\
\text { tradicional foi } \\
\text { consideravelmente reduzida, } \\
\text { praticamente ficando } \\
\text { disponível apenas para } \\
\text { sistemas com capacidade } \\
\text { abaixo de } 100 \text { kW ou por meio } \\
\text { de leilão (licitação). }\end{array}$ \\
\hline \multirow{3}{*}{ Japão } & $\begin{array}{l}\text { Japan’s Residential } \\
\text { PV Dissemination } \\
\text { (RPDV) Program } \\
\text { (1994) }\end{array}$ & $\begin{array}{l}\text { - Instalação de } 250.000 \\
\text { sistemas, totalizando mais de } \\
930 \text { MW (1994-2005) } \\
\text { - Posterior desaceleração após o } \\
\text { fim do programa }\end{array}$ & $\begin{array}{l}\text { - Reembolso previsto de até } 70 \% \\
\text { do custo de instalação dos } \\
\text { sistemas fotovoltaicos }\end{array}$ \\
\hline & $\begin{array}{l}\text { Purchasing Scheme } \\
\text { for Solar PV } \\
\text { Electricity (2009) }\end{array}$ & $\begin{array}{l}\text { - Grande expansão entre } 2008 \text { e } \\
\text { 2011; quanto a capacidade } \\
\text { instalada adicionada em } 2011 \text {, } \\
\text { 85\% do total são residenciais. }\end{array}$ & $\begin{array}{l}\text { - Retomada do RPDV e o início do } \\
\text { programa de compra do } \\
\text { excedente gerado em sistemas } \\
\text { fotovoltaicos (contrato de } 10 \\
\text { anos). } 0 \text { custo incorrido com a } \\
\text { compra dos excedentes foi } \\
\text { repassado por cada } \\
\text { distribuidora aos seus } \\
\text { consumidores (feed-in). }\end{array}$ \\
\hline & $\begin{array}{l}\text { Act on Purchase of } \\
\text { Renewable } \\
\text { Energy Sourced }\end{array}$ & $\begin{array}{l}\text { - Em 2012, aproximadamente } \\
58 \% \text { da capacidade instalada } \\
\text { foi residencial. A partir de } 2013\end{array}$ & $\begin{array}{l}\text { - Incentivo passou a incluir a } \\
\text { compra de energia gerada em } \\
\text { sistemas fotovoltaicos, tendo }\end{array}$ \\
\hline
\end{tabular}




\begin{tabular}{cll}
\hline $\begin{array}{c}\text { Electricity by Electric } \\
\text { Utilities (2012) }\end{array}$ & $\begin{array}{l}\text { houve mudança de política e o } \\
\text { foco passa a ser sistemas de } \\
\text { maior porte. }\end{array}$ & $\begin{array}{l}\text { como foco o desenvolvimento } \\
\text { dos sistemas não residenciais. }\end{array}$ \\
\hline
\end{tabular}

Fonte: DE CASTR0, N.; DANTAS, G., 2018; FEDERAL MINISTRY F0R ECONOMIC AFFAIRS AND ENERGY, 2019.

\section{Análise de Carbono}

Este capítulo tem como objetivo fazer uma análise da emissão de carbono associando-a a atividade de usinas termelétricas e suas respectivas geração histórica ocorrida e projetada.

Comparativamente serão apresentados os cenários de projeções futuras da geração distribuída, a fim de verificar a redução da emissão de gases de efeito estufa (GEE) provenientes da fonte de geração termelétrica brasileira.

A Tabela 3 mostra o panorama atual (fevereiro de 2019) da matriz de geração elétrica brasileira.

Tabela 3. Capacidade de Geração do Brasil.

\begin{tabular}{lcccc}
\hline \multicolumn{1}{c}{ TIP0 } & QUANTIDADE & P0TÊNCIA 0UTORGADA $(\mathbf{k W})$ & POTÊNCIA FISCALIZADA $(\mathbf{k W})$ & \% \\
\hline $\begin{array}{l}\text { Central Geradora } \\
\text { Hidráulica }\end{array}$ & 696 & 698.324 & 695.138 & 0,43 \\
$\begin{array}{l}\text { Central Geradora } \\
\text { Uni-elétrica }\end{array}$ & 1 & 50 & 50 & 0 \\
$\begin{array}{l}\text { Central Geradora } \\
\text { Eólica }\end{array}$ & 589 & 14.562 .239 & 14.516 .793 & 8,91 \\
$\begin{array}{l}\text { Pequena Central } \\
\text { Hidrelétrica }\end{array}$ & 428 & 5.184 .029 & 5.160 .380 & 3,17 \\
$\begin{array}{l}\text { Central Geradora } \\
\text { Solar Fotovoltaica }\end{array}$ & 2.354 & 1.920 .677 & 1.900 .677 & 1,17 \\
$\begin{array}{l}\text { Usina Hidrelétrica } \\
\text { Usina }\end{array}$ & 217 & 101.879 .778 & 98.248 .011 & 60,3 \\
Termelétrica & 3.004 & 42.050 .986 & 40.421 .327 & 24,8 \\
$\begin{array}{l}\text { Usina } \\
\text { Termonuclear }\end{array}$ & 2 & 1.990 .000 & 1.990 .000 & 1,22 \\
Total & 7.291 & 168.286 .083 & 162.932 .376 & 100 \\
\hline Fonte: Agência Naci & & & & \\
\hline
\end{tabular}

Fonte: Agência Nacional de Energia Elétrica, 2019. 
Tendo em vista que o sistema brasileiro é majoritariamente composto por hidrelétricas, as termelétricas funcionam como energia de reserva (energia de partida rápida) para ocasiões como disfunções hidrológicas e aumentos inesperados de demanda. As centrais termelétricas possuem uma participação significativa na matriz brasileira, cerca de 25\% da potência total instalada no Sistema Interligado Nacional (ANEEL, 2018). Porém, as projeções indicam que essa participação diminuirá até 2026 e ficará próxima de 19\% da potência de geração total instalada (EPE, 2017), conforme observado no Gráfico 5, que apresenta o histórico e projeção de capacidade instalada de UTEs com base nos dados de Balanço Energético Nacional (EPE, 2018), Plano Decenal de Expansão de Energia 2026 (EPE, 2017) e Resenha Energética Brasileira (MME, 2018).

Gráfico 5. Histórico e Projeção de Capacidade Instalada de UTEs (MW)

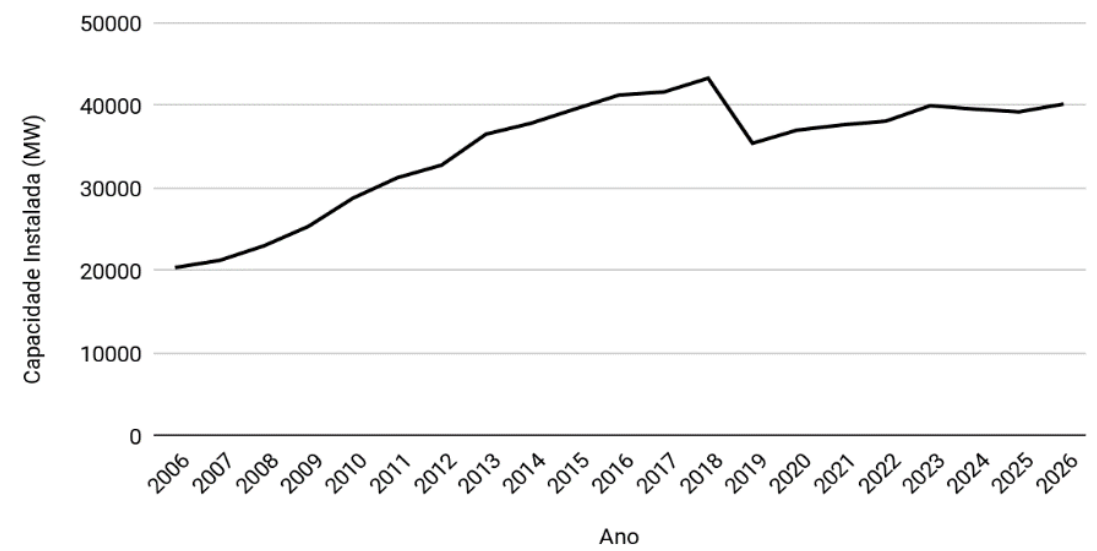

Fonte: Autores.

Entre os anos de 2018 e 2019 houve uma queda significativa na potência total instalada, de 43.303 MW para 35.422 MW. Fato justificado tanto pela não contratação de empreendimentos do tipo, quanto pela desativação de alguns destes. A partir de 2019 a potência instalada volta a crescer até 2023, quando tende a se estabilizar na faixa dos 40.000 MW até 2026. Esta pequena ascensão está relacionada com a contratação de novos empreendimentos, especialmente os vinculados ao gás natural e a biomassa como combustíveis (EPE, 2017).

\section{Redução da Emissão de GEE pela Projeção da Geração Distribuída}

Apesar das projeções otimistas e seu grande potencial de neutralização de carbono, a geração distribuída solar fotovoltaica ainda é incipiente no território nacional. Com a publicação da Resolução Normativa $n^{\circ} 687$ da ANEEL a relação entre distribuidora e “prosumidor” melhorou, de forma que o processo de conexão à rede se tornou mais célere, além do aumento do limite da capacidade do sistema de geração. 
Somam-se a isso outros incentivos para o crescimento a partir de 2015, como (i) a queda dos preços dos painéis fotovoltaicos e maior atratividade de payback; (ii) a mudança de mentalidade da sociedade, convergindo para um pensamento de necessidade de maior sustentabilidade dos processos de geração; (iii) os incentivos nacionais, ainda que sejam insignificantes se comparados às potências na modalidade; e (iv) o crescimento das tarifas de consumo de energia na distribuição.

Foi efetuada a quantificação das emissões de GEE provenientes da geração termelétrica em intervalo de duas décadas (2006-2026) para que, comparativamente, seja possível verificar as tendências de evitação destas emissões com a inserção e crescimento projetado da geração distribuída, segundo o PDE 2026.

Para calcular essa emissão, foram considerados os dados de projeção observados nos (i) Planos Decenais de Expansão da Energia; (ii) em dados passados, oriundos do Balanço Energético Nacional (EPE, 2018); (iii) no Boletim de Monitoramento do Sistema Elétrico Brasileiro (MME, 2018) e na Resenha Energética Brasileira (MME, 2018); (iv) e no fator médio de emissão de $\mathrm{CO}_{2}$ estabelecido pelo Ministério da Ciência e Tecnologia (MCTI, 2018).

Para o cálculo das emissões de $\mathrm{CO}_{2}$ multiplicou-se o fator médio de emissão ( $\mathrm{tCO}_{2} / \mathrm{MWh}$ ) mensal (MCTI, 2018) pela energia elétrica gerada (MWh) pela fonte termelétrica. A projeção das emissões de GEE a partir da fonte termelétrica é evidenciada no Gráfico 6, que expõe as Toneladas de Carbono Equivalente Evitadas com a Inserção da GD na Matriz Energética Brasileira com base nos dados de Balanço Energético Nacional (EPE, 2018), Plano Decenal de Expansão de Energia 2026 (EPE, 2017) e Resenha Energética Brasileira (MME, 2018).

Gráfico 6. Total de Carbono Equivalente gerado pelas UTEs por ano (ktC02eq)

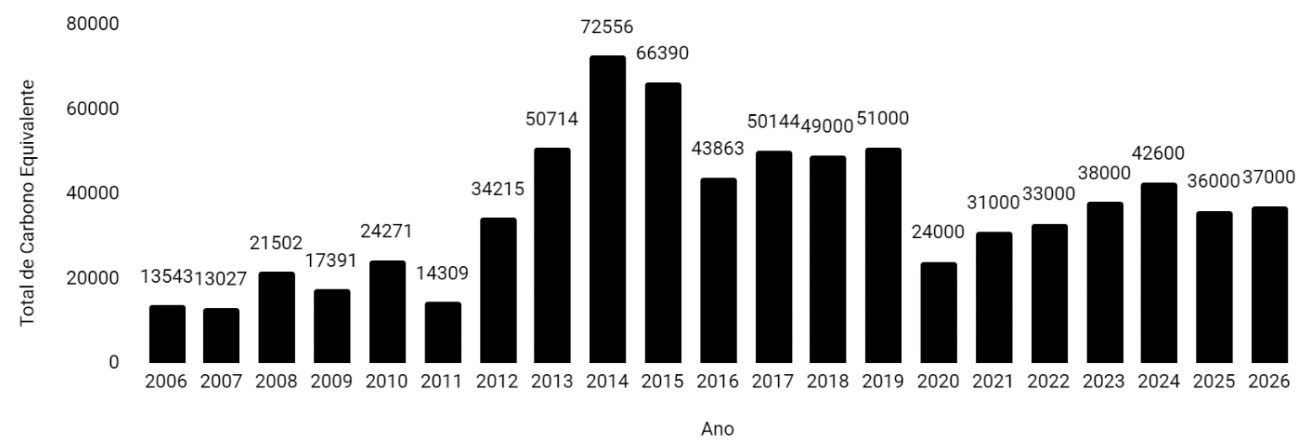

Fonte: Autores.

Observa-se que de 2006 a 2011 os níveis de emissão foram relativamente baixos. A partir de 2011, houve aumento considerável das emissões, com a entrada em operação de empreendimentos termoelétricos em virtude da crise hídrica que afetou o Brasil a partir de 2010. 
Entre 2014 e 2015, com níveis reduzidos nos reservatórios das usinas hidrelétricas, a geração termelétrica foi acionada com maior frequência. 0 resultado refletiu nos níveis de emissão alcançando um pico de 72,5 $\mathrm{MtCO}_{2}$ em 2014. Com o restabelecimento da normalidade do ciclo hídrico, os níveis voltam a reduzir de forma considerável em 2016 e 2017, porém são bem superiores aos registrados no início do período estudado, chegando a $44 \mathrm{MtCO}_{2}$ em 2016 e 50 $\mathrm{MtCO}_{2}$ em 2017. As projeções são otimistas do ponto de vista ambiental, estimando-se que com a inserção das renováveis e a obsolescência de alguns combustíveis fósseis, o nível de emissões caia mais ainda e varie entre 30 e $40 \mathrm{MtCO}_{2}$.

De forma análoga, calculou-se o quantitativo de $\mathrm{CO}_{2}$ equivalente evitado com a projeção da geração distribuída (Gráfico 6), onde: (i) projetou-se para cada ano o incremento de potência instalada de geração distribuída fotovoltaica, conforme os dados do PDE 2026; e (ii) substituiuse a potência instalada da geração termelétrica pela geração distribuída.

\section{RESULTADOS E DISCUSSÃ0}

Para a projeção da mitigação de emissão média para os próximos anos, adotou-se um fator médio ( $\left.\mathrm{tCO}_{2} / \mathrm{MWh}\right)$ do período de 2006 - 2018, correspondente a $0,0683 \mathrm{tCO}_{2} / \mathrm{MWh}$, e posteriormente multiplicou-se este fator pela produção de energia (MWh) a partir da potência total instalada projetada de geração distribuída solar fotovoltaica.

Como resultado foi observado que a evitação da emissão de GEE teórica, em substituição da fonte térmica, pela geração distribuída para 2019 é de aproximadamente 22 mil toneladas de $\mathrm{CO}_{2 e q}$, até 2021 a evitação acumulada é de 67,4 mil toneladas de $\mathrm{CO}_{2 e q}$; e finalmente em 2026 a evitação acumulada é de 337 mil toneladas de $\mathrm{CO}_{2 \text { eq. }}$.

Gráfico 7. Toneladas de $\mathrm{CO}_{2 \mathrm{eq}}$ Evitadas com a Inserção da GD na Matriz Energética Brasileira. 


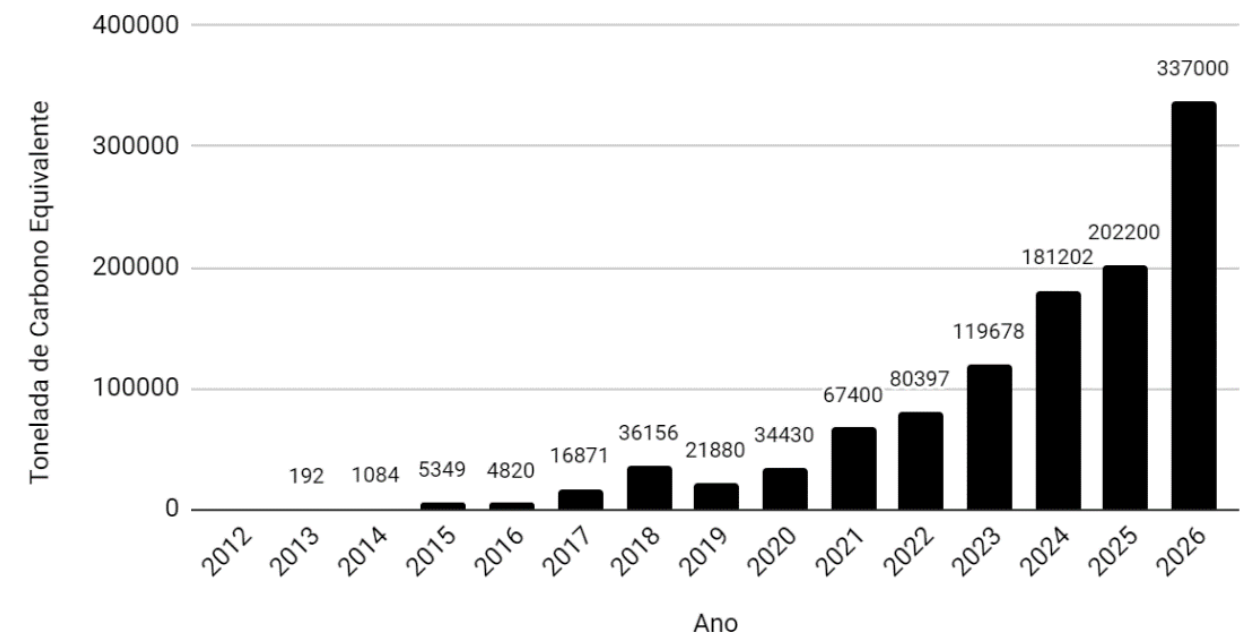

Fonte: Autores.

Com a finalidade de dimensionar espacialmente os resultados de evitação das emissões de $\mathrm{CO}_{2 \text { eq }}$ devido a projeção da GD, foi estimada a quantidade de árvores necessárias e a área que ocupariam para fixar o $\mathrm{CO}_{2 \text { eq }}$ evitado, processo também conhecido como compensação ambiental por fixação de carbono.

Para tal, foram utilizadas as métricas definidas pelo estudo realizado pelo Instituto Totum e pela Escola de Agricultura Luiz de Queiroz (ESALQ) da USP em parceria com a Fundação SOS Mata Atlântica no ano de 2011. No estudo foram adotadas 2 áreas de controle e considerado um plantio médio de 1.667 árvores em uma área de $10.000 \mathrm{~m}^{2}$ (1 árvore a cada $6 \mathrm{~m}^{2}$ ). Foram consideradas árvores nativas de espécies e idades diferentes no bioma da Mata Atlântica.

Para a primeira área de controle, ao longo de 11 anos (2000 a 2011), o plantio de 23.354.266 árvores retirou da atmosfera aproximadamente 1,05 milhão de toneladas de $\mathrm{C}_{2}$ equivalente, ou seja, $4,1 \mathrm{~kg}$ de $\mathrm{CO}_{2 \text { eq }}$ por árvore plantada por ano, durante o período de 11 anos do projeto da primeira área de controle. Na segunda área de controle, onde ao longo de 8 anos (2003 a 2011) foram plantadas 3.842.426 árvores, foram sequestradas 194,23 mil toneladas de $\mathrm{CO}_{2 \mathrm{eq}}$, o que corresponde à remoção de $6,32 \mathrm{~kg}$ de $\mathrm{CO}_{2 \text { eq }}$ por árvore por ano, considerando o período de 8 anos do projeto da segunda área de controle. Considerando o cenário mais conservador, foi adotada uma fixação média de $4,1 \mathrm{~kg}$ de $\mathrm{CO}_{2 \text { eq }}$ por árvore plantada por ano.

Portanto, o estudo estima que cada árvore da Mata Atlântica absorve em média $82 \mathrm{~kg}$ de $\mathrm{CO}_{2}$ equivalente ao longo de seus primeiros 20 anos ou ainda que são necessárias 12,2 árvores nativas de Mata Atlântica para compensar cada tonelada de $\mathrm{CO}_{2 \mathrm{eq}}$ emitida.

Considerando esta métrica e o espaçamento de plantio de árvores de $6 \mathrm{~m}^{2}$ a cada indivíduo plantado, pode-se dimensionar a área necessária para compensação de 337.000 ton de $\mathrm{CO}_{2 \text { eq, }}$ o que equivale a 4.111 .400 árvores nativas de Mata Atlântica em 2.466,35 hectares ou 
24,66 km². Tal plantio corresponde à evitação das emissões de GEE em 2026 pelo estabelecimento da geração distribuída solar fotovoltaica calculada. A área de 24,66 km² corresponde a 56 vezes o território do Vaticano.

\section{Detalhamento}

Em análise às políticas de incentivo dos países estudados e aos resultados obtidos, podese notar uma tendência em relação a implantação das tarifas feed-in e geração de crédito fiscal para adquirir os sistemas fotovoltaicos de pequena escala, estas converteram em resultados satisfatórios. É possível identificar, nas experiências internacionais, que os países que adotaram a política de financiamentos a juros baixos não obtiveram resultados expressivos.

No Brasil, pode-se observar que as políticas de incentivo que regulamentam créditos na conta de luz do consumidor/gerador e a isenção de pagamento de impostos, assim como a criação do Fundo Clima e do ProGD, fizeram com que o país tivesse uma projeção otimista dentro do PDE 2026, superadas no ano de 2018, mostrando assim o resultado positivo destas políticas de incentivo.

Quanto à análise de emissões de GEE, a partir dos resultados alcançados, é possível notar a tendência de minimização das emissões a partir da difusão da geração distribuída e redução da participação da geração de energia termelétrica de 28,82\% em 2013, de 25,3\% em 2018, e uma projeção de 18,9\% em 2026. Esta variação é justificada pela obsolescência, não contratação de novos empreendimentos e desmobilização de antigas geradoras à base de combustíveis fósseis.

A redução nas emissões identificada nos últimos anos vai ao encontro das metas firmadas no Acordo de Paris em 2016 em relação à produção de energia elétrica, as quais consideram, reduzir as emissões de carbono baseadas no ano de 2005 em 37\% até 2025 e em 43\% até 2030. Com base nessas informações, cabe destacar que a geração distribuída de energia através dos sistemas fotovoltaicos é uma das formas de neutralização de carbono.

\section{CONCLUSÃ0}

É possível identificar que o Brasil está seguindo as tendências internacionais, com a queda dos preços dos sistemas e financiamentos mais consistentes para compra destes. 0 Brasil encontra desafios para expansão da GD, como a regulamentação adequada às singularidades regionais e formas de incentivo que se mostraram mais efetivas no cenário internacional.

É interessante a inserção de políticas de incentivo locais em menor escala como feito no Japão e na Alemanha no início do desenvolvimento da geração distribuída solar fotovoltaica, 
através da atuação municipal, por exemplo, visando criar uma relação mais próxima com possíveis interessados em adquirir essa tecnologia. No estudo dos países de referência é evidente que as tarifas feed-in são alicerces de impulsão da geração distribuída, bem como os subsídios governamentais no processo de instalação e na compra dos painéis.

0 desenvolvimento da tecnologia nacional dos equipamentos, qualificação da mão de obra especializada, e a redução da tributação aos insumos dos componentes das placas, têm potencial para tornar os custos de implantação menores a partir da nacionalização da cadeia.

Por sua característica continental, o Brasil, assim como a China, enfrenta dificuldades para aproximar a geração do consumo, já que o maior centro de consumo de carga se encontra no eixo Sul/Sudeste brasileiro; e as maiores usinas geradoras de energia localizam-se no Norte/Nordeste do país, por suas características naturais favoráveis para base de geração energética brasileira. Deste modo, a geração distribuída surge como alternativa para aproximar o consumo e a geração, minimizando-se assim os custos, impactos e riscos associados à transmissão de energia.

Os esforços no sentido de expandir a geração distribuída na matriz energética brasileira trazem benefícios claros, como a mitigação das emissões de GEE associadas à operação de usinas termelétricas. A partir do incremento de potência instalada de 3,6 GW de GD fotovoltaica no horizonte decenal foi estimada a evitação acumulada de 337 mil toneladas de $\mathrm{CO}_{2 \text { eq, }}$, correspondente a uma área de reflorestamento de aproximadamente 25.000 .000 de $\mathrm{m}^{2}$ área correspondente a 56 vezes o território do Vaticano. Esta mensuração torna palpável as vantagens relacionadas à diversificação da matriz elétrica brasileira em prol de fontes renováveis.

A partir destas recomendações, busca-se a promoção de um modelo mais limpo e sustentável, a fim de reduzir a geração de carbono e fazer com que a GD se dissemine pelo país. Como resultado, espera-se o desenvolvimento de uma matriz elétrica complementar e diversa, que garanta confiabilidade do sistema e melhor aproveitamento do potencial elétrico brasileiro.

\section{REFERÊNCIAS BIBLIOGRÁFICAS}

ACKERMANN, Thomas et al. Distributed generation: a definition. Eletric Power Systems Research, [S.l.], p. 195-204, jan. 2001. Disponível em: 〈https://www.sciencedirect.com/science/article/abs/pii/S0378779601001018〉. Acesso em: 27 ago. 2018.

AGÊNCIA NACIONAL DE ENERGIA ELÉTRICA. (Brasil). Matriz de Energia Elétrica. Disponível em: 〈http://www2.aneel.gov.br/aplicacoes/capacidadebrasil/OperacaoCapacidadeBrasil.cfm>. Acesso em: 30 jun. 2019. 
AGÊNCIA NACIONAL DE ENERGIA ELÉTRICA. (Brasil). Resolução Normativa $\mathbf{n}^{0}$ 482: Estabelece as condições gerais para 0 acesso de microgeração e minigeração distribuída aos sistemas de distribuição de energia elétrica, 0 sistema de compensação de energia elétrica, e dá outras providências. [S.l.: s.n.], 2012. 1-12 p. Disponível em:

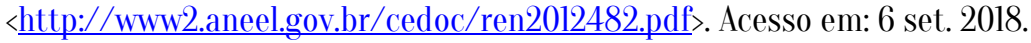

AGÊNCIA NACIONAL DE ENERGIA ELÉTRICA. (Brasil). Resolução Normativa no 687.[S.l.: s.n.], 2015. 1-25 p. Disponível em: 〈http://www2.aneel.gov.br/cedoc/ren2015687.pdf $>$.

Acesso em: 3 set. 2018.

ASSOCIAÇ̃̃o BRASILEIRA DE ENERGIA SOLAR FOTOVOLTAICA (ABSOLAR). Infográfico ABSOLAR. Brasil: [s.n.], 2017. Disponivel em: $<$ http://www.absolar.org.br/infografico-absolar.html〉. Acesso em: 25 ago. 2020

DE CASTR0, Nivalde; DANTAS, Guilherme (Org.). Experiências Internacionais em Geração em Distribuída: Motivações, Impactos e Ajustes. Rio de Janeiro: Publit Soluções Editoriais, 2018. 1-442 p.

DE CASTR0, Nivalde; DANTAS, Guilherme (0rg.). Geração distribuída: Experiências Internacionais e análises comparadas. Grupo de Estudos do Setor Elétrico, p. 200, Disponível em:

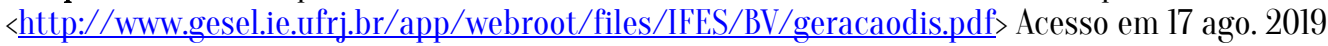

EMPRESA DE PESQUISA ENERGÉTICA. Balanço Energético Nacional. Brasil: [s.n.], 2013. Disponível em:

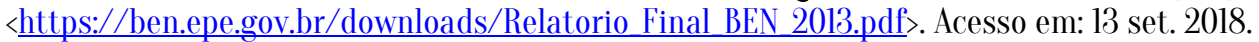

EMPRESA DE PESQUISA ENERGÉTICA. (Brasil). Plano Decenal de Expansão de Energia 2026. Brasil: [s.n.], 2017. 1-271 p. Disponível em: $\quad$ http://www.epe.gov.br/sites-pt/publicacoes-dadosabertos/publicacoes/PublicacoesArquivos/publicacao-40/PDE2026.pdf >. Acesso em: 28 set. 2018.

FEDERAL MINISTRY FOR ECONOMIC AFFAIRS AND ENERGY (Alemanha). Time series for the development of renewable energy sources in Germany. Alemanha: Federal Ministry for Economic Affairs and Energy, Agosto 2019. Disponivel em: <https://www.erneuerbare-energien.de/EE/Redaktion/DE/Downloads/zeitreihen-zur-entwicklungder-erneuerbaren-energien-in-deutschland-1990-2018-en.pdf? _blob=publicationFileøvV=7>. Acesso em: 27 set. 2019 .

FRANZ, Büro F (2016). Regulatory Trends in Renewable Energy Self-Supply - A Summary of International Debates. GIZ. Santiago do Chile, fevereiro de 2016.

HERNÁNDEZ-CALLEJO, Luis; GALLARDO-SAAVEDRA, Sandra; ALONSO-GÓMES; Victor. A review of photovoltaic systems: Design, operation and maintenance. Solar Energy, [S. l.], p. 426-440, 1 ago. 2019. Disponível em: 〈https://doi.org/10.1016/j.solener.2019.06.017〉. Acesso em: 21 jul. 2019.

INTERNATIONAL ENERGY AGENCY. Renewables 2018. [S. I.], 2018. Disponível em: 〈https://www.iea.org/renewables2018/power/〉. Acesso em: 19 jul. 2019.

INTERNATIONAL ENERGY AGENCY (IEA). Snapshot Of Global Photovoltaic Markets. ISBN 978-3-906042-72-5. 2018.

MINISTÉRIO DA CIÊNCIA, TECNOLOGIA, INOVAÇÕES E COMUNICAÇÕES. (Brasil). Fator médio - Inventários $\begin{array}{lllll}\text { corporativos. } & \text { Brasil: } & \text { [s.n.], } & 2018 . & \text { Disponível }\end{array}$ 〈http://www.mctic.gov.br/mctic/opencms/ciencia/SEPED/clima/textogeral/emissao_corporativos.html〉. Acesso em: 10 set. 2018.

MINISTÉRI0 DE MINAS E ENERGIA. (Brasil). Boletim de Monitoramento do Sistema Elétrico Brasileiro. Brasil: [s.n.], [2009 - 2018]. 1 - final p. Disponivel em: 〈http://www.mme.gov.br/web/guest/secretarias/energiaeletrica/publicacoes/boletim-de-monitoramento-do-sistema-eletrico>. Acesso em: 12 set. 2018.

MINISTÉRI0 DE MINAS E ENERGIA. Resenha Energética Brasileira. Disponível em: < http://www.mme.gov.br/documents/36208/948169/Resenha+Energ\%C3 \%A9tica+Brasileira++edi\%C3\%A7\%C3\%A30+2019+v3.pdf/92ed2633- e412-d064-6ael-eefac950168b>, Acesso em: 12 set. 2018.

PESTANA, Daniel Garigali; RODRIGUES, Sandy; MORGAD0-DIAS, F. Environmental and economic analysis of solar systems in Madeira, Portugal. Utilities Policy, [S. l.], p. 31-40, 3 set. 2018. 
SE4ALL, Global tracking framework: overview. World Bank Report for Sustainable Energy for All. 2014. Disponível em: 〈http://www.worldbank.org/en/topic/energy/publication/Global-Tracking-Framework-Report. >. Acesso em: 1 fev. 2019.

SOS MATA ATLÂNTICA. Cada árvore da Mata Atlântica chega a tirar 163 kg de gás carbônico da atmosfera. Brasil, 2011. Disponível em: 〈https://www.sosma.org.br/13135/cada-arvore-da-mata-atlantica-chega-a-retirar-163-kg-degas-carbonico-da-atmosfera/>. Acesso em: 25 jan. 2019.

ZHANG, Lei; QIN, Quande; WEI, Yi-Ming. China's distributed energy policies: Evolution, instruments and recommendation. Energy Policy 125, [S.l.], p. 55-64, jan. 2019. Disponível em: 〈https://www.journals.elsevier.com/energy-policy . Acesso em: 16 jan. 2019. 